Pacific Journal of Mathematics

CENTRAL EMBEDDINGS IN SEMI-SIMPLE RINGS 


\section{CENTRAL EMBEDDINGS IN SEMI-SIMPLE RINGS}

\section{S. A. Amitsur}

A ring $S$ is a central extension of a subring $R$ if $S=R C$ and $C$ is the centralizer of $R$ in $S$, i.e., $C=\{s \in S ; s r=r s\}$ for every $r \in R$. We shall also say that $R$ is centrally embedded in $S$.

We have shown that if a ring $R$ is centrally embedded in a simple artinian ring then $R$ is a prime Öre ring and its quotient ring $Q$ is the minimal central extension of $R$ which is a simple artinian ring; furthermore, the centralizer of $R$ can be characterized. In the present note we extend these results and show that rings which can be centrally embedded in semi-simple artinian rings are semi-prime Öre rings with a finite number of minimal primes and their rings of quotients are the minimal central extension of this type.

2. The Ring $Q_{0}(R)$. We recall some definitions and results of [1].

Let $R$ be an associative ring (not necessarily with a unit) and let $L_{0}(R)$ be the set of all (two-sided) ideals $A$ of $R$ with the property:

(A) " $\forall x \in R, A x=0 \Rightarrow x=0 "$.

The set $L_{0}(R)$ is a filter. That is: closed under finite intersection and inclusion. We shall also. assume henceforth that $R \in L_{0}(R)$ i.e. $R x=0 \Rightarrow x=0$.

Consider every $A \in L_{0}(R)$ as left $R$-module and define the ring $Q_{0}(R)=\lim _{\rightarrow} \operatorname{Hom}_{R}(A, R)$, where $A$ ranges over all $A \in L_{0}(R)$. A more detailed description of $Q_{0}(R)$ is as follows: Let $U=\cup \operatorname{Hom}_{R}(A, R)$, $A \in L_{0}(R)$, and in $U$ we define an equivalence relation, addition and multiplication as follows:

For $\alpha: A \rightarrow R, \beta: B \rightarrow R$ and $A, B \in L_{0}(R)$ we put:

(i) $\alpha+\beta: A \cap B \rightarrow R$ defined by $x(\alpha+\beta)=x \alpha+x \beta$ for $x \in A \cap B$.

(ii) $\alpha \beta: B A \rightarrow R$ by: $(\Sigma b a) \alpha \beta=\Sigma[b(a \alpha)] \beta$ for $b \in B, a \in A$.

(iii) $\alpha \equiv \beta$ if there exists $C \subseteq A \cap B, C \in L_{0}(R)$ for which $c \alpha=$ $c \beta$ for every $c \in C$.

The ring $Q_{0}(R)$ is the ring of equivalence classes of $U$ with respect to preceding definitions. Furthermore, $R$ is canonically mapped into $Q_{0}(R)$ by identifying $R$ with the right multiplications on $R$.

The center $\Gamma=\Gamma(R)$ of $Q_{0}(R)$ can be characterized as the set of all $\bar{\gamma} \in Q_{0}(R)$ which have a representative $\gamma \in \operatorname{Hom}(A, R)$ such that $\gamma$ is in 
fact a bi-R-module homomorphism of $A$ into $R$. i.e. it satisfies $(a x) \gamma=$ $(a \gamma) x$ and $(x a) \gamma=x(a \gamma)$ for $a \in A, x \in R$. Also $\bar{\gamma} \in \Gamma$ if and only if it commutes with the element of $R$.

From the results of [1] we quote the following:

If $R$ is semi-simple artinian, then $R$ is both a right and left Öre ring and its quotient ring is $Q_{0}(R)=R \Gamma$. [1, Theorem 6].

If $S=R C$ is a simple artinian central extension of $R$ then $\Gamma \subseteq C$, $S=R \Gamma \otimes_{\Gamma} C$ and $R \Gamma=Q_{0}(R)$ is also simple artinian [1. Theorem 18].

The ring $R \Gamma$ is semi-simple artinian if and only if the number of minimal primes $P$ of $R$ is finite, and for each $P,(R / P) \Gamma(R / P)$ is simple artinian. [1, Corollary 13].

It follows also from the proofs of [1, Theorem 10] that the number of simple components of $R$ equals the number of minimal primes of $R$.

3. The main result. Let $S=S_{1} \oplus \cdots \oplus S_{m}$ a direct sum of a finite number of simple rings $S_{i}$ with units $\epsilon_{i}$, and $1=$ $\epsilon_{1}+\epsilon_{2}+\cdots+\epsilon_{n}$. The ring $S$ will be said an extension of minimal length of a subring $R$ if for every $i$ there exist $0 \neq r \in R$ such that $r \epsilon_{j}=0$ for all $j \neq i$, or equivalently $r\left(1-\epsilon_{i}\right)=0$. This means that for no subring $S\left(1-\epsilon_{i}\right)=S_{1} \oplus \cdots \oplus S_{i-1} \oplus S_{i+1} \oplus \cdots \oplus S_{m}$ the subring $R\left(1-\epsilon_{i}\right)$ is isomorphic with $R$.

Lemma 1. Let $S=R C$ be a central extension of $R$. and let $S=S_{1} \oplus \cdots \oplus S_{m}$ be a direct sum of simple rings $S_{i}$ with units $\epsilon_{i}$. Then :

(1) For every central idempotent $\epsilon, S \epsilon$ is a central extension of $R \epsilon$; and it is also a direct sum of simple rings with a unit.

(2) There exists a direct summand $S \epsilon$ of $S$ such that $R \cong R \epsilon$, and $S \epsilon$ is a central extension of $R$ of minimal length.

Proof. A central idempotent $\epsilon$ of $S$ is of the form $\epsilon=\epsilon_{i 1}+\cdots+\epsilon_{i r}$ and hence $S \epsilon=S_{i_{1}} \oplus S_{i_{2}} \oplus \cdots \oplus S_{i_{r}}$. Furthermore $S=R C$ yields that $S \epsilon=(R C) \epsilon=(R \epsilon)(C \epsilon)$ and the elements of $C \epsilon$ commute with the elements of $C \epsilon$, which readily implies that $S \epsilon$ is a central extension of $R \epsilon$.

To prove the second part, we consider the set of all central idempotents $\epsilon$ of $S$ with the property: " $r \epsilon=0, r \in R \Rightarrow r=$ 0". Clearly for such $\epsilon, R \cong R \epsilon$ by corresponding: $r \rightarrow r \epsilon$. The set of these idempotents is not empty since the unit 1 has this property. Each of the central idempotent $\epsilon$ has the form $\epsilon=\epsilon_{i_{1}}+\cdots \epsilon_{i_{o}}, i_{1}<i_{2}<\cdots<$ $i_{r}$. So choose $\epsilon$ of this set with minimal $\rho$. Then $S \epsilon$ is a central extension of $R \epsilon$ of minimal length, since the minimality of $\rho$ implies that for any $1 \leqq \lambda \leqq \rho$, there exists $r \neq 0$ such that $r\left(\epsilon-\epsilon_{i_{\lambda}}\right)=0$.

The preceding lemma shows that if a ring $R$ has a central extension 
$S$ of the type described above, then replacing $S$ by a direct summand we get a central extension of minimal length of a ring isomorphic with $R$. We can, therefore, restrict ourselves to the study of central extension of minimal length. Our results is the following.

THEOREM A. Let $S=R C$ be a central extension of $R$ of minimal length then $R$ is semi-prime and we can embed $\Gamma \subseteq C$. Furthermore, $R \Gamma$ is also a central extension of $R$ of the same type with the same number of components.

THEOREM B. Let $S=R C$ be a semi-simple artinian ring and a central extension of $R$ of minimal length then $R=Q_{0}(R)$ is also semi-simple artinian and $S=R \Gamma \otimes_{\Gamma} C$.

In view of the results quoted from [1] we deduce that:

COROllary $C$. If $R$ has a central extension which is a semi-simple artinian ring, then $R$ is a semi-prime (right and left) Öre ring with a finite number of minimal primes. Its ring of quotient is $Q_{0}(R)$ and it is a minimal semi-simple artinian central extension of $R$.

4. Proofs. Before proceeding with the proof we need a few lemmas.

Lemma 2. Let $S=R C$ be a central extension of $R$ of minimal length, then an ideal $A$ in $R$ belongs to $L_{0}(R)$ if and only if $A C=S$.

Indeed, let $S=S_{1} \oplus \cdots \oplus S_{n}, S_{i}$ simple with a unit $\epsilon_{i}$. If $A C=S$ and $A x=0$ for some $x \in R$, then $S x=(A C) x=(A x) C=0$ but $S$ has a unit and so $x=0$, i.e. $A \in L_{0}(R)$. Conversely, it suffices to show that $A C \cap S_{i} \neq 0$, since then $A C \cap S_{i}$ is a nonzero ideal in the simple ring implies that $S_{i}=A C \cap S_{i}$. This in turn yields that $A C \supset S_{i}$ and, therefore $A C \supset S_{1} \oplus \cdots \oplus S_{n}=S$. To prove that $A C \cap S_{i} \neq 0$, we note that if $A C \cap S_{i}=0$ then $A \epsilon_{i} \subseteq A S_{i} \subseteq A R C \cap S_{i} \subseteq A C \cap S_{i}=0$. Let $P=\left\{r, r \epsilon_{i}=0\right\}$ and $Q=\left\{r \in R, r\left(1-\epsilon_{i}\right)=0\right\}$. Since $S$ is of minimal length it follows that $P \cap Q=0, Q \neq 0$ and $P \supseteq A$. Thus $A Q \subseteq$ $P \cap Q=0$ which contradicts the assumption that $A \in L_{0}(R)$ (i.e.. $A$ satisfies (A) of $\$ 2$ ).

We can follow now the proofs of [1] Lemma 14 and show:

LEMMA 3. If $S$ is as above then there is an embedding of $\Gamma$ into the center of $S$ which contains $C$.

Proof. Let $\alpha: A \rightarrow R, A \in L_{0}(R)$ be a representative of an element $\bar{\alpha} \in \Gamma$. First we show that there is a unique element $c_{\alpha} \in C$ 
depending on $\bar{\alpha}$ (and not on the representative $\alpha$ ) such that $a \alpha=a c_{\alpha}$ for every $a \in A$. Next we prove that the correspondence: $\bar{\alpha} \rightarrow \delta_{\alpha}$ is the required embedding. The proof follows the proof of [1] Lemma 14.

Since $A \in L_{0}(R)$, it follows by Lemma 2 that $A C=S$ and hence $1=\Sigma a_{i} c_{i}$ for some $a_{i} \in A$ and $c_{i} \in C$. Set $c_{\alpha}=\Sigma\left(a_{i} \alpha\right) c_{i}$. Since $\bar{\alpha} \in \Gamma$. $\alpha$ is a bi- $R$ hence for every $a \in A$ :

$$
a \alpha=(a \alpha) 1=\Sigma(a \alpha) a_{i} c_{\imath}=\Sigma\left(a \alpha_{\imath}\right) \alpha c_{\imath}=a \Sigma\left(a_{i} \alpha\right) c_{i}=a c_{\alpha} .
$$

To prove that $c_{\alpha} \in C$, we observe that for every $a \in A$ and $x \in R:(a x) c_{\alpha}=(a x) \alpha=(a \alpha) x=a c_{\alpha} x$. Hence, $\quad a\left(x c_{\alpha}-c_{\alpha} x\right)=$ 0. Consequently, $S\left(x c_{\alpha}-c_{\alpha} x\right)=(C A)\left(x c_{\alpha}-c_{\alpha} x\right)=0$ and since $1 \in S$ it follows that $x c_{\alpha}-c_{\alpha} x=0$ for every $x \in R$, i.e. $c_{\alpha} \in C$.

The element $c_{\alpha}$ which belongs to $C$, actually commutes also with the elements of $R$ and hence belongs to the center of $S$. Indeed, let $c \in C$ and $a \in A$ then since $C$ centralizes $A$ we have $(a \alpha) c=c(a \alpha)$ as $a \alpha \in R$. Also $a \alpha=a c_{\alpha}=c_{\alpha} a$ and, therefore:

$$
c_{\alpha}(a c)=\left(a c_{\alpha}\right) c=(a \alpha) c=c(a \alpha)=(c a) c_{\alpha}=(a c) c_{\alpha} .
$$

That is, $c_{\alpha}$ commutes with all the elements of $A C=S$, and this means that $c_{\alpha}$ is in the center of $S$.

Next we show that $c_{\alpha}$ depends only on $\bar{\alpha} \in F$ : let $\beta: B \rightarrow R$ be another representative of $\bar{\alpha}$ then $\alpha=\beta$ on some $D \subseteq A \cap B$ which belongs to $L_{0}(R)$. Hence for $d \in D: d c_{\alpha}=d \alpha=d \beta=d c_{\beta}$. which implies that $D\left(c_{\alpha}-c_{\beta}\right)=0$ and therefore $S\left(c_{\alpha}-c_{\beta}\right)=(C D)\left(c_{\alpha}-c_{\beta}\right)=0$ which yields $c_{\alpha}-c_{\beta}=0$.

Finally $c_{\alpha+\beta}=c_{\alpha}+c_{\beta}, c_{\alpha \beta}=c_{\alpha} c_{\beta}$ since for some ideals in $L_{0}(R)$ we have the following relations for their elements:

$$
\begin{aligned}
x c_{\alpha+\beta} & =x(\alpha+\beta)=x \alpha+x \beta=x c_{\alpha}+x c_{\beta}=x\left(c_{\alpha}+c_{\beta}\right) \\
y c_{\alpha \beta} & =y(\alpha \beta)=(y \alpha) \beta=(y \alpha) c_{\beta}=y\left(c_{\alpha} c_{\beta}\right)
\end{aligned}
$$

and as in preceding proofs this implies that $c_{\alpha+\beta}=c_{\alpha}+c_{\beta}$ and $c_{\alpha \beta}=c_{\alpha} c_{\beta}$.

We, henceforth, identify $\Gamma$ with its image in $C$ and thus we may assume that $\Gamma \subseteq C$.

Lemma 4. Let $S=R C=S_{1} \oplus \cdots \oplus S_{n}$, $S_{1}$ simple with unit $\epsilon_{l}$, be a central extension of $R$ of minimal type, then $\epsilon_{i} \in \Gamma$.

For let $P=\left\{r \in R, r \epsilon_{i}=0\right\}$ and $Q=\left\{r \in R, r\left(1-\epsilon_{i}\right)=0\right\}$. Since $S$ of minimal length, $P \neq 0, Q \neq 0$ and $P \cap Q-0$. We first assert that $P+Q \in L_{0}(R)$ and, indeed, $(Q C) \epsilon_{i}=\left(Q \epsilon_{i}\right) C=Q C=Q R C=Q S=$ 
$Q \neq 0$ and so $Q C \subseteq S_{i}$ but $Q C$ is and ideal in $S$ and therefore, also in $S_{\text {, }}$ which yields $Q C=S_{i}$ since $S_{i}$ is simple. A similar proof which uses the fact that $P \epsilon_{j} \neq 0$ for $j \neq i$ shows that $(P C) \epsilon_{j}=S_{j}$. Hence

$$
(P+Q) C=\Sigma(P+Q) C_{k}=\Sigma S_{k}=S
$$

and thus $P+Q \in L_{0}(R)$ by Lemma 1. Consider now the map $\epsilon$ : $P+Q \rightarrow Q$ given by $(p+q) \epsilon=q$. Clearly, this is a bi- $R-$ homomorphism, hence $\bar{\alpha} \in \Gamma$ and so there exists $c_{\epsilon} \in C$ such that $(p+q) c_{\epsilon}=q$. Consequently, $(p+q) c_{\epsilon}=q=q \epsilon_{l}=(p+q) \epsilon_{i}$. By the uniqueness of $c_{\epsilon}$ it follows that $c_{\epsilon}=\epsilon_{i}$

We are now in position to prove the main theorems.

$R$ is semi-prime, for if $A^{2}=0$ then $(A C)^{2}=$ in $S$, but $S$ is semiprime and so $A C=0$ which implies that $A=0$.

Let $S=R C=S_{1} \oplus \cdots \oplus S_{n}$ be a central extension of $R$ of minimal length, with $\epsilon_{i}$ the units of $S_{i}$. Put $P=\left\{r \in R, r \epsilon_{1}=0\right\}$, and consider $R$ as a subring of $Q_{0}(R)$. Then we readily have, since $\epsilon_{1} \in \Gamma \subseteq Q_{0}(R)$ that $P=R \cap Q_{0}(R)\left(1-\epsilon_{1}\right)$. Furthermore, $P$ is a prime ideal: indeed let $A B \subseteq P$ with $A, B$ ideals in $R$ containing $P$, then since $B \not \subset P, B \epsilon_{1} \neq 0$ and, therefore, $(B C) \epsilon_{1}$ is a nonzero ideal in $S_{1}$ which implies that $B C \epsilon_{1}=S_{1}$. Thus:

$$
0=(C P) \epsilon_{1} \supseteq(C A B) \epsilon_{1}=A(C B) \epsilon_{1}=A S_{1} .
$$

This yields that $A \epsilon_{1}=0$ and so $A \subseteq P$. We can now apply [1] Theorem 8 , which in our case means that $Q_{0}(R / P) \cong Q_{0}(R) \epsilon_{1}$ and $\Gamma(R / P) \cong$ $\Gamma(R) \epsilon_{1}=\Gamma \epsilon_{1}$.

Denote, $R_{1}=R \epsilon_{1}$ (which isomorphic with $R / P$ ) and $c_{1}=c \epsilon_{1}$ then $R C \epsilon_{1}=R_{1} C_{1}=S_{1}$ which shows that $R_{1}$ is a prime ring with a central extension which is a simple ring $S_{1}$ with a unit. It follows, therefore, by [1] Theorem 18 that $R_{1} \Gamma\left(R_{1}\right)$ is simple with a unit. Now $\Gamma\left(R_{1}\right)=$ $\Gamma(R / P)=\Gamma \epsilon_{1}$ by the preceding result. So $R_{1}\left(\Gamma \epsilon_{1}\right)$ is simple with a unit and note also that $R_{1} \Gamma \epsilon_{1}=(R \Gamma) \epsilon_{1}$. The same follows for all the other idempotents $\epsilon_{i}$ and so we get that $R \Gamma=R \Gamma \epsilon_{1}+R \Gamma \epsilon_{2}+\cdots+R \Gamma \epsilon_{n}$ is a direct sum of simple rings with units, which completes the proof of Theorem A.

The proof of Theorem B follows the same lines by applying the second part of [1] Theorem 18 which was quoted in the present note (\$2). Namely, if $S$ is semi-simple artinian then each summand $S_{i}$ is simple artinian and hence, by [1] Theorem $18 R_{1} \Gamma_{1}=\left(R \epsilon_{1}\right)\left(\Gamma \epsilon_{1}\right)=(R \Gamma) \epsilon_{1}$ is simple artinian. Furthermore, we also have $R_{1} \Gamma_{1}=Q_{0}(R / P)=$ $Q_{0}(R) \epsilon_{1}$ by (iii) of [1] Theorem B. Thus, $Q_{0}(R)=\Sigma Q_{0}(R) \epsilon_{i}=\Sigma R_{i} \Gamma_{i}=$ $R \Gamma$. 
Finally, $(R C) \epsilon_{i}=R_{\imath} \Gamma_{1} \otimes_{\Gamma_{1}} C \epsilon_{i}$ for every $i$, from which it follows that:

$$
R C=\Sigma R C \epsilon_{i}=\Sigma R \Gamma_{i} \underset{\Gamma_{i}}{\otimes} C \epsilon_{i} \cong R \Gamma \underset{\Gamma}{\otimes} C
$$

since $\Gamma=\Sigma \Gamma \epsilon_{i}$ and the elements $\epsilon_{i}$ belong to the center of $S=R C$. The last isomorphism is given by the mappings $r \alpha \otimes c \rightarrow \Sigma(r \alpha) \epsilon_{i} \otimes_{\Gamma_{1}} c \epsilon_{i}$; $r \alpha_{i} \otimes_{r_{1}} c \epsilon_{i} \rightarrow r \alpha_{i} \otimes_{r} c \epsilon_{i}$.

Corollary $\mathrm{C}$ follows now immediately by Theorem 6 and Corollary 13 of [1].

We finish with an immediate corollary of the fact that $\Gamma \subseteq$ Cents $S$, and Cent $S \subseteq C$ :

COROllary D. If $R C$ is a central embedding of $R$ in a direct sum of simple rings of minimal length, then so is $R($ Cent $C)$.

\section{REFERENCES}

1. S. A. Amitsur, On rings of quotients, Instituto Nazionale di Alta Matematica Symposia Matematica v. VIII (1972), 149-164.

Received December 28, 1973.

The Hebrew University of Jerusalem 



\section{Pacific Journal of Mathematics}

\section{Vol. 56, No. $1 \quad$ November, 1975}

Shimshon A. Amitsur, Central embeddings in semi-simple rings .......... 1

David Marion Arnold and Charles Estep Murley, Abelian groups, A, such

that $\operatorname{Hom}(A,---)$ preserves direct sums of copies of $A \ldots \ldots \ldots .$.

Martin Bartelt, An integral representation for strictly continuous linear

operators ................................... 21

Richard G. Burton, Fractional elements in multiplicative lattices......... 35

James Alan Cochran, Growth estimates for the singular values of

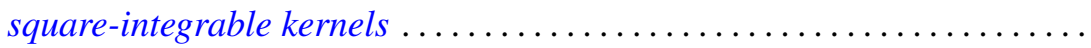

C. Martin Edwards and Peter John Stacey, On group algebras of central

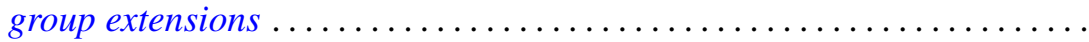

Peter Fletcher and Pei Liu, Topologies compatible with homeomorphism

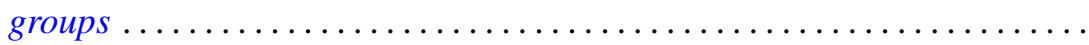

George Gasper, Jr., Products of terminating ${ }_{3} F_{2}(1)$ series ............ 87

Leon Gerber, The orthocentric simplex as an extreme simplex ............

Burrell Washington Helton, A product integral solution of a Riccati



Melvyn W. Jeter, On the extremal elements of the convex cone of

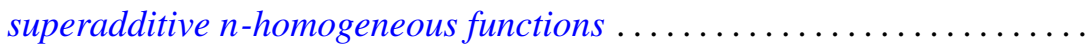

R. H. Johnson, Simple separable graphs .

Margaret Humm Kleinfeld, More on a generalization of commutative and

alternative rings. . .

A. Y. W. Lau, The boundary of a semilattice on an $n$-cell.

Robert F. Lax, The local rigidity of the moduli scheme for curves ...

Glenn Richard Luecke, A note on quasidiagonal and quasitriangular

operators .

Paul Milnes, On the extension of continuous and almost periodic functions

Hidegoro Nakano and Kazumi Nakano, Connector theory.

James Michael Osterburg, Completely outer Galois theory of perfect rings ..................................

Lavon Barry Page, Compact Hankel operators and the F. and M. Riesz

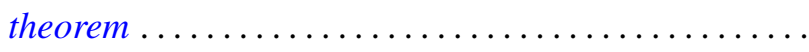

Joseph E. Quinn, Intermediate Riesz spaces..................... 225

Shlomo Vinner, Model-completeness in a first order language with a generalized quantifier.

Jorge Viola-Prioli, On absolutely torsion-free rings ..........

Philip William Walker, A note on differential equations with all solutions of integrable-square............................

Stephen Jeffrey Willson, Equivariant maps between representation 